NBER WORKING PAPER SERIES

\title{
MACHINE LEARNING FOR REGULARIZED SURVEY FORECAST COMBINATION: PARTIALLY-EGALITARIAN LASSO AND ITS DERIVATIVES
}

Francis X. Diebold

Minchul Shin

Working Paper 24967

http://www.nber.org/papers/w24967

\author{
NATIONAL BUREAU OF ECONOMIC RESEARCH \\ 1050 Massachusetts Avenue \\ Cambridge, MA 02138 \\ August 2018
}

This is a revised and extended version of our earlier-circulated manuscript, "Beating the Simple Average: Egalitarian LASSO for Combining Economic Forecasts". For comments we are grateful to the editors (Domenico Giannone, George Kapetanios, and Mike McCracken), two anonymous referees, and Umut Akovali, Xu Cheng, Denis Chetverikov, Edgar Dobriban, Ed George, Mike Kearns, Laura Liu, Ken McAlinn, Rob McCulloch, Hashem Pesaran, Veronika Rockova, Zhentao Shi, Tara Sinclair, Stephen Stigler, Dongho Song, Allan Timmermann, Weijie Su, Jonathan Wright, and Boyuan Zhang. The paper also benefitted from presentations at FRB St. Louis, Brown, Chicago, TU Vienna, York, the ECB, and the NBER. The usual disclaimer applies. The views expressed herein are those of the authors and do not necessarily reflect the views of the National Bureau of Economic Research.

NBER working papers are circulated for discussion and comment purposes. They have not been peer-reviewed or been subject to the review by the NBER Board of Directors that accompanies official NBER publications.

(C) 2018 by Francis X. Diebold and Minchul Shin. All rights reserved. Short sections of text, not to exceed two paragraphs, may be quoted without explicit permission provided that full credit, including $\odot$ notice, is given to the source. 
Machine Learning for Regularized Survey Forecast Combination: Partially-Egalitarian Lasso and its Derivatives

Francis X. Diebold and Minchul Shin

NBER Working Paper No. 24967

August 2018

JEL No. C53

\begin{abstract}
Despite the clear success of forecast combination in many economic environments, several important issues remain incompletely resolved. The issues relate to selection of the set of forecasts to combine, and whether some form of additional regularization (e.g., shrinkage) is desirable. Against this background, and also considering the frequently-found good performance of simple-average combinations, we propose a LASSO-based procedure that sets some combining weights to zero and shrinks the survivors toward equality ("partially-egalitarian LASSO"). Ex-post analysis reveals that the optimal solution has a very simple form: The vast majority of forecasters should be discarded, and the remainder should be averaged. We therefore propose and explore direct subset-averaging procedures motivated by the structure of partiallyegalitarian LASSO and the lessons learned, which, unlike LASSO, do not require choice of a tuning parameter. Intriguingly, in an application to the European Central Bank Survey of Professional Forecasters, our procedures outperform simple average and median forecasts indeed they perform approximately as well as the ex-post best forecaster.
\end{abstract}

Francis X. Diebold

Department of Economics

University of Pennsylvania

133 South 36th Street

Philadelphia, PA 19104-6297

and NBER

fdiebold@ sas.upenn.edu

Minchul Shin

Department of Economics

University of Illinois

214 David Kinley Hall

1407 W. Gregory

Urbana, IL 61801

mincshin@illinois.edu 


\section{Introduction}

Forecast combination has a long and successful history in economics. ${ }^{1}$ Important issues remain incompletely resolved, however, related to determining the best set of forecasts to combine ("selection", e.g., via an information criterion), how to combine those selected (e.g., via a linear weighted average), and whether some form of regularization (e.g., via shrinkage) is desirable given that the historical forecast record is often small relative to the number of candidate forecasters. Against this background, and also considering the frequentlyfound good performance of simple-average combinations, we propose various LASSO-inspired procedures that address all considerations.

We proceed as follows. In section 2, we highlight aspects of the "equal-weights puzzle", that is, the frequently-found good performance of simple-average combinations, which motivates our concerns and proposals, and we describe our "partially-egalitarian LASSO" procedures, which shrink and select in desirable ways. In section 3 we provide ex post empirical assessment of our procedure's performance. In section 4 we propose and explore direct ex ante combination procedures motivated by the structure of partially-egalitarian LASSO and the lessons learned. In section 5 we place our methods in the context of the broader literature, which notably includes Capistrán and Timmermann (2009), Elliott (2011), Conflitti et al. (2015), and Samuels and Sekkel (2017), among many others. We conclude in section 6.

\section{Partially-Egalitarian LASSO for Forecast Combination}

In this section we consider methods for selection and shrinkage in regression-based forecast combination. The key new method is "partially-egalitarian LASSO" (peLASSO). We build to it gradually, arriving at peLASSO in section 2.6.

\subsection{Aspects of Optimal Forecast Combination}

Although it seems natural to average forecasts (i.e., to use equal-weight combinations), simple averages are generally suboptimal. To see the theoretical sub-optimality of equal combining weights, consider $K$ competing unbiased forecasts $f_{t}^{1}, \ldots, f_{t}^{K}$ of $y_{t}$. We form a combined

\footnotetext{
${ }^{1}$ For overviews see Diebold and Lopez (1996), Timmermann (2006), and Elliott and Timmermann (2016).
} 
forecast as

$$
C_{t}=\beta_{1} f_{t}^{1}+\beta_{2} f_{t}^{2}+\ldots+\left(1-\sum_{k=1}^{K-1} \beta_{i}\right) f_{t}^{K} .
$$

The corresponding forecast errors, $e_{C t}$ and $e_{1 t}, \ldots, e_{K t}$, have variances $\sigma_{C}^{2}$ and $\sigma_{1}^{2}, \ldots, \sigma_{K}^{2}$, and they satisfy the same equality, from which it follows that the variance of the combined forecast error is minimized using the weight vector

$$
\beta^{*}=\left(\Sigma^{-1} \mathbf{i}\right) /\left(\mathbf{i}^{\prime} \Sigma^{-1} \mathbf{i}\right)
$$

where $\Sigma$ is the variance-covariance matrix of the forecast errors and $\mathbf{i}$ is a conformable column vector of ones (Bates and Granger (1969)). In particular, equal weights - that is, simple averages - are generally suboptimal. ${ }^{2}$

It is well known (Granger and Ramanathan (1984)) that the population Bates-Granger optimal combining weights (1) introduced above may be trivially obtained from the population regression (linear projection) $y_{t} \rightarrow f_{t}^{1}, \ldots, f_{t}^{K}$, subject to the constraint that the coefficients add to one. ${ }^{3}$ So the theoretical optimal linear forecast combination problem is just a population linear regression (projection) problem, and finite-sample combining weight estimation involves just a simple linear regression.

Despite the theoretical sub-optimality of equal weights, a large literature finds frequent good performance of simple averages under quadratic loss. Indeed the forecast combination "equal weights puzzle", emphasized long ago by Clemen (1989) and Diebold (1989), refers to the frequently-found good performance of simple averages. ${ }^{4}$ The equal weights puzzle has by now been well-studied and is better understood. Aruoba et al. (2012), for example, work in population (i.e., without estimation error) and show that: (1) even if simple averages are not fully optimal, they are likely to be much better than any individual forecast, and (2) even if simple averages are not fully optimal, they are likely to be close to the optimum. In

${ }^{2}$ As an example, consider two forecasts with uncorrelated errors. Then (1) reduces to

$$
\beta^{*}=\frac{\sigma_{2}^{2}}{\sigma_{1}^{2}+\sigma_{2}^{2}}=\frac{1}{1+\phi^{2}},
$$

where $\beta^{*}$ is the weight placed on forecast 1 and $\phi=\sigma_{1} / \sigma_{2}$. Hence the simple average obtains if and only if $\phi=1$. This is entirely natural - we want to give more weight to the forecast with lower-variance errors, so we take a simple average only in the equal-variance case.

${ }^{3}$ Moreover, one can allow for biased forecasts by including an intercept, and there is no real need to impose the "sum-to-one" constraint.

${ }^{4}$ Note well that the theoretical suboptimality of simple averages, and hence the equal weights puzzle, refers to combination under quadratic loss. Under other loss functions, equal weights may in fact be optimal. Aruoba et al. (2012), for example, show that equal weights are optimal under minimax loss. 
addition, Smith and Wallis (2009) show that finite-sample combining-weight estimation error can seriously degrade empirical attempts at optimal combination, which further increases the relative attractiveness of simple averages, since they do not involve estimation.

The discussion thus far strongly suggests that simple averages (equal weights) are a natural shrinkage direction for such combining regressions. With shrinkage, we don't force simple averages; rather, we coax things in that direction, blending data (likelihood) information with prior information. This amounts to a Bayesian approach with the prior centered on simple averages.

An important issue remains, however. Particularly when combining large numbers of forecasts, some forecasts may be largely redundant, or not worth including in the combination for a variety of other reasons. So we potentially want to set some combining weights to zero ("select to zero") and shrink the remaining weights toward equality ("shrink toward equality"). As we will see, LASSO-based methods almost do the trick - they both select and shrink - but unfortunately they select to zero and shrink to zero. In the remainder of this section we begin by expositing standard LASSO, which we then modify until we arrive at our "partially-egalitarian LASSO", which selects to zero and shrinks to equality. Interestingly, each of the estimators introduced en route will prove useful in its implementation. ${ }^{5}$

\subsection{Penalized Estimation for Selection and Shrinkage}

Consider a penalized forecast combining regression, with "parameter budget" $c$,

$$
\hat{\beta}_{\text {Penalized }}=\arg \min _{\beta} \sum_{t=1}^{T}\left(y_{t}-\sum_{i=1}^{K} \beta_{i} f_{i t}\right)^{2} \text { s.t. } \sum_{i=1}^{K}\left|\beta_{i}\right|^{q} \leq c .
$$

Equivalently, in Lagrange-multiplier form we can write

$$
\hat{\beta}_{\text {Penalized }}=\arg \min _{\beta}\left(\sum_{t=1}^{T}\left(y_{t}-\sum_{i=1}^{K} \beta_{i} f_{i t}\right)^{2}+\lambda \sum_{i=1}^{K}\left|\beta_{i}\right|^{q}\right)
$$

where $\lambda$ depends on $c$. Taking $\lambda=0$ produces Bates-Granger OLS combining:

$$
\hat{\beta}_{B G}=\arg \min _{\beta} \sum_{t=1}^{T}\left(y_{t}-\sum_{i=1}^{K} \beta_{i} f_{i t}\right)^{2} .
$$

\footnotetext{
${ }^{5}$ For a broad introduction to LASSO and related procedures, see Hastie et al. (2009).
} 
Many estimators that select and/or shrink, both of which are important for our purposes, fit in the penalized estimation framework. ${ }^{6}$

\subsection{Shrinkage Toward Equality: Egalitarian Ridge}

Smooth convex penalties in (2) produce pure shrinkage. In particular, $q=2$ produces Ridge regression, which shrinks coefficients toward 0 :

$$
\hat{\beta}_{\text {Ridge }}=\arg \min _{\beta}\left(\sum_{t=1}^{T}\left(y_{t}-\sum_{i=1}^{K} \beta_{i} f_{i t}\right)^{2}+\lambda \sum_{i=1}^{K} \beta_{i}{ }^{2}\right) .
$$

Taking $q=2$ and centering the constraint around $1 / K$ produces a modified Ridge regression that shrinks coefficients toward equality ("egalitarian ridge", or "eRidge"):

$$
\hat{\beta}_{\text {eRidge }}=\arg \min _{\beta}\left(\sum_{t=1}^{T}\left(y_{t}-\sum_{i=1}^{K} \beta_{i} f_{i t}\right)^{2}+\lambda \sum_{i=1}^{K}\left(\beta_{i}-\frac{1}{K}\right)^{2}\right) .
$$

eRidge is closely related to the Bayesian shrinkage combining weight estimation of Diebold and Pauly (1990), who take an empirical Bayes approach using the $g$-prior of Zellner (1986), but it is simpler to implement.

Note that, although eRidge will feature later in this paper (which is why we introduced it), it is inadequate for our ultimate purpose - it shrinks in the right direction but does not select.

\subsection{Selection to and Shrinkage Toward Zero: LASSO}

As we have noted, $q=2$ produces pure shrinkage (Ridge). Conversely, $q \rightarrow 0$ produces pure selection. The intermediate case $q=1$ produces shrinkage and selection and is known as a LASSO estimator:

\footnotetext{
${ }^{6}$ One could also add additional constraints. For example, with unbiased forecasts it may be natural to impose $\beta_{i} \geq 0, \forall i$ and $\sum_{i=1}^{K} \beta_{i}=1$, as in Conflitti et al. (2015), but we will not pursue that here.
} 


$$
\hat{\beta}_{L A S S O}=\arg \min _{\beta}\left(\sum_{t=1}^{T}\left(y_{t}-\sum_{i=1}^{K} \beta_{i} f_{i t}\right)^{2}+\lambda \sum_{i=1}^{K}\left|\beta_{i}\right|\right) .
$$

The seminal reference is Tibshirani (1996).

There are several variants of LASSO. The most important for our purposes is "adaptive LASSO" (Zou (2006)), which weights the terms in the penalty to encourage setting small first-round coefficient estimates to zero,

$$
\hat{\beta}_{a L A S S O}=\arg \min _{\beta}\left(\sum_{t=1}^{T}\left(y_{t}-\sum_{i=1}^{K} \beta_{i} f_{i t}\right)^{2}+\lambda \sum_{i=1}^{K} w_{i}\left|\beta_{i}\right|\right)
$$

where $w_{i}=1 /\left|\hat{\beta}_{i}\right|^{\nu}, \hat{\beta}_{i}$ is OLS or ridge, and $\nu>0$. Others include "elastic net" (Zou and Hastie (2005)), which uses a convex combination of the LASSO $(q=1)$ and ridge penalties $(q=2)$, namely $\sum_{i=1}^{K}\left(\alpha\left|\beta_{i}\right|+(1-\alpha) \beta_{i}^{2}\right)$, and "adaptive elastic net", which blends the adaptive LASSO and elastic net penalties as $\sum_{i=1}^{K}\left(\alpha w_{i}\left|\beta_{i}\right|+(1-\alpha) \beta_{i}^{2}\right)$.

Under some assumptions, the adaptive versions (adaptive LASSO and adaptive elastic net) have the so-called "oracle property". 7 The elastic net variants have good properties in handling highly-correlated predictors. Adaptive elastic net has both. Unfortunately, however, all LASSO variants, although improving on ridge insofar as they shrink and select, remain inadequate for our purposes - they select in the right direction (to zero) but shrink in the wrong direction (toward zero).

\subsection{Selection to and Shrinkage Toward Equality: Egalitarian LASSO}

All of the standard LASSO variants in section 2.4 select and shrink combining weights toward zero, but that is not what we want. Instead, as discussed in section 2.1, both theory and experience point clearly to shrinkage toward simple averages. We therefore change the LASSO penalized estimation problem to

$$
\hat{\beta}_{e L A S S O}=\arg \min _{\beta}\left(\sum_{t=1}^{T}\left(y_{t}-\sum_{i=1}^{K} \beta_{i} f_{i t}\right)^{2}+\lambda \sum_{i=1}^{K}\left|\beta_{i}-\frac{1}{K}\right|\right) .
$$

\footnotetext{
${ }^{7}$ That is, roughly put, they asymptotically select the data-generating process (DGP) almost surely if it is among the models considered, and they otherwise select the best predictive approximation to the DGP.
} 
That is, instead of shrinking the weights toward zero, we shrink the deviations from equal weights toward zero. In Appendix A we show that eLASSO implementation is straightforward using standard software.

Note that although eLASSO shrinks in the right direction, it is still unappealing, for reasons opposite those of standard LASSO. Like standard LASSO, eLASSO shrinks and selects, but whereas LASSO shrinks in the wrong direction, eLASSO selects in the wrong direction! We nevertheless introduced Ridge, eRidge, LASSO, and eLASSO because the procedure to which we now turn - which shrinks and selects in the right direction - is closely related, and because each will feature importantly in our subsequent empirical work.

\subsection{Selection to Zero and Shrinkage Toward Equality: Partially-Egalitarian LASSO}

eLASSO does not tend to discard forecasters, because it selects and shrinks toward equal weights, not toward zero weights. In particular, eLASSO implicitly presumes that all forecasters "belong" in the set to be combined. One can easily modify the eLASSO, however, such that some forecasters are potentially discarded, and then the survivors are selected and shrunken toward equality. We call this "partially-egalitarian LASSO".

\subsubsection{1-Step Conceptualization}

Partially-egalitarian LASSO (peLASSO) solves a penalized estimation problem with two penalties,

$$
\hat{\beta}_{p e L A S S O}=\arg \min _{\beta}\left(\sum_{t=1}^{T}\left(y_{t}-\sum_{i=1}^{K} \beta_{i} f_{i t}\right)^{2}+\lambda_{1} \sum_{i=1}^{K}\left|\beta_{i}\right|+\lambda_{2} \sum_{i=1}^{K}\left|\beta_{i}-\frac{1}{p(\beta)}\right|\right),
$$

where $p(\beta)$ is the number of non-zero elements in $\beta$. The first is the standard LASSO penalty, selecting and shrinking to zero, whereas the second selects and shrinks to equality. Optimization of this 1-step objective proves difficult, due to discontinuity of the objective function at $\beta_{i}=0$. We therefore reserve it to future work, proceeding instead with a 2-step approach.

\subsubsection{2-Step Implementation}

The obvious 2-step analog of equation (3) above is: 
Step 1 (Select to Zero): Using standard methods, select $k$ forecasts from among the full set of $K$ forecasts.

Step 2 (Shrink Toward Equality): Using standard methods, shrink the combining weights on the $k$ forecasts that survive step 1 toward $1 / k$.

The obvious method for step 1 is standard LASSO, which requires only one estimation and moreover can handle situations with $K>T$, which are not uncommon in forecast combination. In our subsequent empirical work, for example, such situations are omnipresent, as our combining regressions involve more forecasters than observations.

One obvious method for step 2 is eRidge, which can be trivially implemented via a standard Ridge regression with a transformed left-hand-side variable, as discussed in Appendix A. One can go even farther and use eLASSO for step 2, in which case the complete procedure would first select some weights to 0 , and then select some of the surviving weights to $1 / k$ and shrink the rest toward $1 / k$.

In the empirical work of sections 3 and 4 below, we emphasize combining procedures motivated by 2-step peLASSO.

\section{$3 \quad$ Ex Post Optimal peLASSO Tuning}

In this section we begin our empirical work, providing a comparative assessment of various forecast combination methods using the European Central Bank's well-known quarterly Survey of Professional Forecasters. ${ }^{8}$ Of course the comparative performance of our methods, using a particular dataset and a particular implementation (choice of sample period, choice of tuning parameters, etc.), cannot establish anything conclusively, but it illustrates our methods in a realistic and important environment, and it provides suggestive evidence regarding their performance.

We emphasize that in this section, for those procedures that require selection of a tuning parameter $\lambda$, we examine out-of-sample RMSE based on the ex-post optimal $\lambda$, i.e., the $\lambda$ that optimizes out-of-sample RMSE had we been using it in real time, which we can determine ex post. ${ }^{9}$ Hence we endow the forecaster with valuable information not available ex ante. Subsequently in section 4 we will show how to address the tuning issue ex ante, the key to which is first understanding the nature of the ex-post solution, to which we now turn.

\footnotetext{
${ }^{8}$ See http://www.ecb.europa.eu/stats/prices/indic/forecast/html/index.en.html.

${ }^{9} \lambda$ could of course be a vector, as with peLASSO.
} 


\subsection{Background}

Again, we focus on the European Central Bank's well-known quarterly Survey of Professional Forecasters. We consider quarterly 1-year-ahead forecasts of Euro-area real GDP growth (year-on-year percentage change). However, as noted by Genre et al. (2013), forecasts are solicited for one year ahead of the latest available outcome. For example, in the 2007Q1 survey, respondents were asked to forecast GDP growth over 2006Q3-2007Q3. Hence our "one-year-ahead" growth forecasts are actually six to eight months ahead.

We have an unbalanced panel, because forecasters enter and exit in real time, and moreover, those in the panel at any time do not necessarily respond to the survey. Hence for ease of analysis we select the 23 forecasters who responded most frequently to the surveys (1999Q1-2016Q2), and we impute missing observations using a linear filter as in Genre et al. (2013). We start with the 1999Q1 survey because the survey began then, and we end with the 2016Q2 survey to ensure that all our growth realizations data are of final revised form, as we now explain.

Throughout we calculate forecast errors using "realizations" from the 2018Q1 data vintage (pulled 2018M5, when the latest revision of this paper was begun, containing what we will consider to be final-revised data for quarters through 2016Q4). The first release of 2016Q4 GDP was in 2017M2, and then it went through several revisions. The statistical agency, Eurostat, makes all "standard" revisions by 100 days after the end of the quarter ("preliminary" 30 days after, "flash" 45 days after, "regular" 60 days after, and "updated" 100 days after), but additional non-standard revisions sometimes occur after more than 100 days, so we wait approximately a year, using "realizations" from the 2018Q1 vintage, to ensure that all realizations are approximately "final-revised" values, which is desirable because forecasters should be forecasting true GDP growth, the best estimate of which is the final-revised value, not a preliminary release.

We do the forecast evaluation as follows. Our surveys run 1999Q1-2016Q2, corresponding to growth rate forecasts running 1999Q3-2016Q4. We burn in our estimation using the first five forecasts 1999Q3-2000Q3, so our actual evaluation period is 2000Q4-2016Q4. We roll through the evaluation sample, estimating combining weights using a 5-year (20-quarter) window and producing a 1-year-ahead out-of-sample forecast. For periods 6-20 we simply estimate using all available data from time 1, despite that fact that there are fewer than 20 observations. ${ }^{10}$ For periods $t>20$ we use a full 20-period estimation window.

We focus on combining methods that involve regularization estimators, which is essential

\footnotetext{
${ }^{10}$ We do this so as not to have to discard the first 20 observations, as degrees of freedom are scarce.
} 
in our context as $K>T$. Our main comparison involves combined forecasts based on Ridge, LASSO, eRidge, eLASSO, and three versions of peLASSO (the first step is always LASSO, and the second step is either simple average, eRidge, or eLASSO). ${ }^{11}$ Throughout, we compare the formally-combined forecasts to simple averages.

Each combining method except simple averages requires choosing a tuning parameter, $\lambda$, which governs regularization strength. We examine combined forecast accuracy for many $\lambda$ 's, ranging from very light penalization ( $\operatorname{small} \lambda$; all forecasters included in the combination) to very heavy penalization (large $\lambda$; no forecasters included in the combination). Specifically, we compute forecasts on a grid of $200 \lambda$ 's. We start with an equally-spaced grid on [-15, $15]$, which we then exponentiate, producing a grid on $(0,3269017]$, with grid coarseness increasing with $\lambda$. This grid turns out to be adequate for all LASSO-based combinations that we consider.

\subsection{Ex Post Results}

We present out-of-sample combined forecast RMSE's in Table 1. There are many relevant observations. In no particular order:

1. Granger-Ramanathan OLS combination is infeasible, because $K>T$, so we cannot include it in the table.

2. No method performs better than the best individual forecaster. (It can happen that a combined forecast is better than any individual forecast, but it doesn't happen here.)

3. All methods perform better than the worst individual forecaster.

4. The simple average improves significantly over the worst individual, but it is still noticeably worse than the best individual.

5. All procedures involving selection to zero select a very small number of forecasters on average (approximately three).

6. Ridge and LASSO perform about as well as the simple average, despite their shrinking toward zero weights rather than equal weights.

\footnotetext{
${ }^{11}$ Unlike much of the LASSO literature, we do not standardize our data. Standardization is desirable when the regressors are measured in different units, but that is not the case in forecast combination, so there is no need.
} 
Table 1: Forecast RMSE's Based on Ex-Post Optimal $\lambda$ 's

\begin{tabular}{lccccc}
\hline \hline Regularization Group & RMSE & $\lambda^{*}$ & $\#$ & DM & p-val \\
\hline Ridge & 1.51 & 2.66 & 23.00 & -0.14 & 0.56 \\
LASSO & 1.52 & 0.38 & 2.71 & -0.10 & 0.54 \\
\hline eRidge & 1.50 & $\max$ & 23.00 & -1.14 & 0.87 \\
eLASSO & 1.50 & 3.60 & 23.00 & 0.95 & 0.17 \\
\hline peLASSO (LASSO, Average) & 1.40 & 0.21 & 2.95 & 1.06 & 0.15 \\
peLASSO (LASSO, eRidge) & 1.40 & $(0.21, \max )$ & 2.95 & 1.06 & 0.15 \\
peLASSO (LASSO, eLASSO) & 1.40 & $(0.21,3.10)$ & 2.95 & 1.07 & 0.15 \\
\hline \hline Comparisons & RMSE & $\lambda^{*}$ & $\#$ & DM & p-val \\
\hline Best & 1.40 & $\mathrm{~N} / \mathrm{A}$ & 1 & 0.61 & 0.27 \\
90\% & 1.44 & $\mathrm{~N} / \mathrm{A}$ & 1 & 0.63 & 0.27 \\
Median & 1.53 & $\mathrm{~N} / \mathrm{A}$ & 1 & -0.57 & 0.72 \\
$10 \%$ & 1.68 & $\mathrm{~N} / \mathrm{A}$ & 1 & -1.61 & 0.94 \\
Worst & 1.74 & $\mathrm{~N} / \mathrm{A}$ & 1 & -1.55 & 0.94 \\
\hline Average & 1.50 & $\mathrm{~N} / \mathrm{A}$ & 23 & $\mathrm{~N} / \mathrm{A}$ & $\mathrm{N} / \mathrm{A}$ \\
\hline \hline
\end{tabular}

Notes: $\lambda^{*}$ is the ex-post optimal penalty parameter(s), \# is the average number of forecasters selected, and DM is the one-sided Diebold and Mariano (1995) statistic against a simple average, with p-value denoted p-val. We compute DM as in Harvey et al. (1999). 
7. eRidge and eLASSO perform exactly as well as the simple average. This is because the optimal regularization (toward the average) turns out to be very strong, in which case both eRidge and eLASSO produce a simple average.

8. All peLASSO methods perform identically. The reason is as follows. They regularize identically in the first step, by construction (all use standard LASSO in step 1). Then, in the second step, the "LASSO, Average" method averages by construction, and the remaining methods effectively average as well in the second step, because heavy step-2 regularization turns out to be optimal.

9. The peLASSO methods reduce out-of-sample RMSE relative to the simple average by almost ten percent.

10. The peLASSO methods have out-of-sample RMSE as good as that of the best forecaster. This property is reminiscent of procedures that achieve external regret minimization in the "combining expert advice" problem, as discussed for example in Arora et al. (2012).

The nature of the ex post optimal solution is contained in results 5 and 8: First discard most forecasters (result 5) and then simply average the survivors (result 8). The importance of this "trim and average" solution cannot be over-emphasized, and we will indeed emphasize and explore it extensively in Section 4.

In Appendix B we show that the results are robust to doing the evaluation only over periods $t>20$, so that we always have an exact 20-period estimation window. In Appendix $\mathrm{C}$ we show that the results are robust (in fact even better) when using aLASSO rather than LASSO in the 2-step peLASSO. The trim-and-average nature of the ex post optimal peLASSO solution remains intact throughout: First discard most forecasters, and then average the survivors.

\subsection{On the Importance of $\lambda$}

The results in Table 1 depend on knowledge of the ex post optimal $\lambda$. To get a feel for the sensitivity to $\lambda$, we show RMSE as function of $\lambda$ in Figure 1. In each sub-figure, the lighter gray line is the RMSE for simple averaging. Consider first the top row of Figure 1, in which we show standard Ridge and standard LASSO. They perform similarly in terms of the optimized value based on the ex post best $\lambda$; at that point they are basically indistinguishable from each other and from a simple average. In the limit as penalization increases, 
Figure 1: RMSE as a Function of $\lambda$, Various Forecast Combination Methods

Standard Ridge

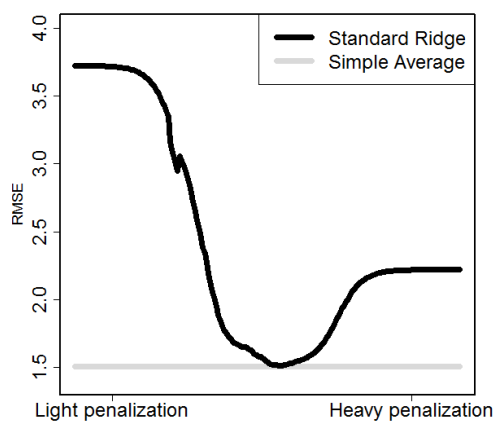

eRidge

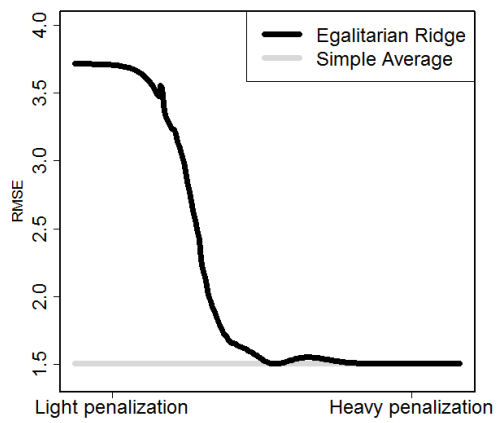

peLASSO

(LASSO in Step 1)

(Simple Average in Step 2)

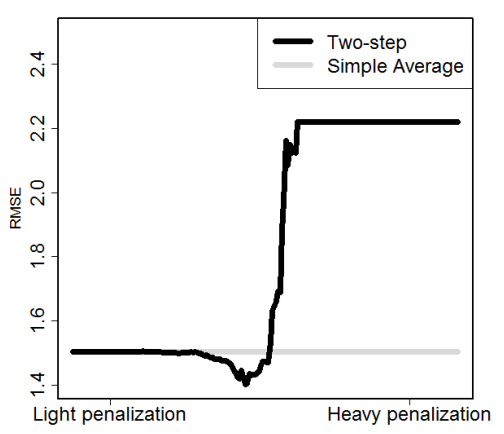

Standard LASSO

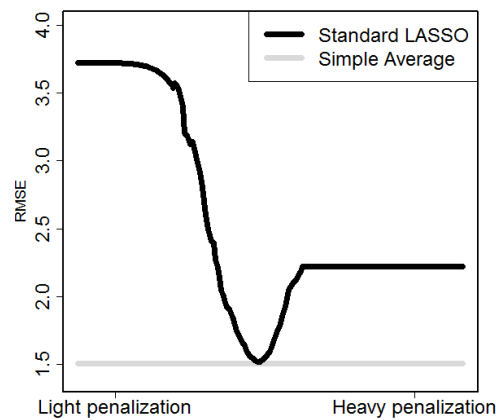

eLASSO

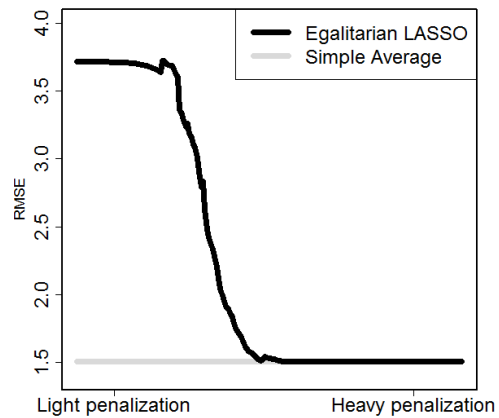

peLASSO

(Optimized LASSO in Step 1) (eLASSO in Step 2)

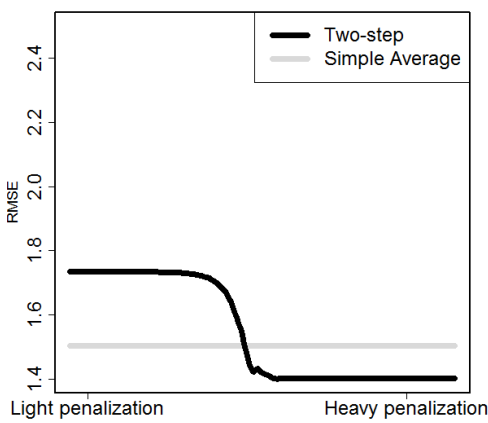

Notes: In the lower-right panel we implement step 2 by egalitarian LASSO regression on the step- 1 selected forecasters, so there is an additional penalty parameter. We show RMSE as a function of the step-2 penalty, with the step-1 penalty fixed at its optimal value. 
however, their performance deteriorates as all forecasters are eventually excluded and the "combined forecasts" therefore approach 0. Finally note that the simple average is never beaten, including at the ex post optimum $\lambda$ 's.

Next consider the second row of Figure 1, in which we show eRidge and eLASSO. They too perform similarly in terms of the optimized value based on the ex post best $\lambda$; at that point they are basically indistinguishable from each other and from a simple average. But their penalization limit is very different. In the limit as penalization increases, eRidge, eLASSO, and simple averaging must be (and are) identical. As in the first row of Figure 1, however, the simple average is never beaten.

Now consider the third row of Figure 1, in which we show peLASSO, in each case with step 1 done by standard LASSO. In the left panel we implement step 2 by simply averaging the step-1 selected forecasters, so there is only one penalty parameter to choose. At the ex post optimum penalty, this 2-step egalitarian LASSO outperforms other methods, including simple averaging of all forecasters.

In the right panel of the third row of Figure 1 we implement step 2 by eLASSO regression on the forecasters selected in step 1, so there is a second penalty parameter to choose. Denote the ex post optimal pair by $\left(\lambda_{1}^{*}, \lambda_{2}^{*}\right)$. We show RMSE as a function of $\lambda_{2}$, with $\lambda_{1}$ fixed at $\lambda_{1}^{*}$. It turns out that once we select forecasters, it is ex post optimal to shrink those selected strongly toward a simple average; that is, heavy step-2 penalization (large $\lambda_{2}$ ) is optimal.

The key result is that unlike other methods (rows 1 and 2 of Figure 1), peLASSO methods (row 3 of Figure 1) offer at least the possibility of beating the simple average. In the remainder of this paper we explore various strategies for attaining the ex post theoretical peLASSO gains in ex ante peLASSO practice.

\subsection{On the Set of Selected Forecasters}

One might wonder about the nature and evolution of the set of forecasters selected by our peLASSO procedures. The selected forecasters are identical across the procedures, periodby-period, because the first step is always the same (LASSO). We show them in Figure 2, as we roll through the sample. The $\mathrm{x}$-axis denotes time, and the y-axis denotes forecaster ranking, where a smaller y-axis location refers to a forecaster with smaller overall RMSE. A "+" symbol at location $(x, y)$ indicates that forecaster $y$ was selected at time $x$.

A number of results emerge. First, The selected set is usually small, with three or four forecasters (as also mentioned earlier in conjunction with Table 1), yet also usually "democratic" in the sense that it is composed of some ex-post top performers, some ex-post 
Figure 2: Selected Forecasters

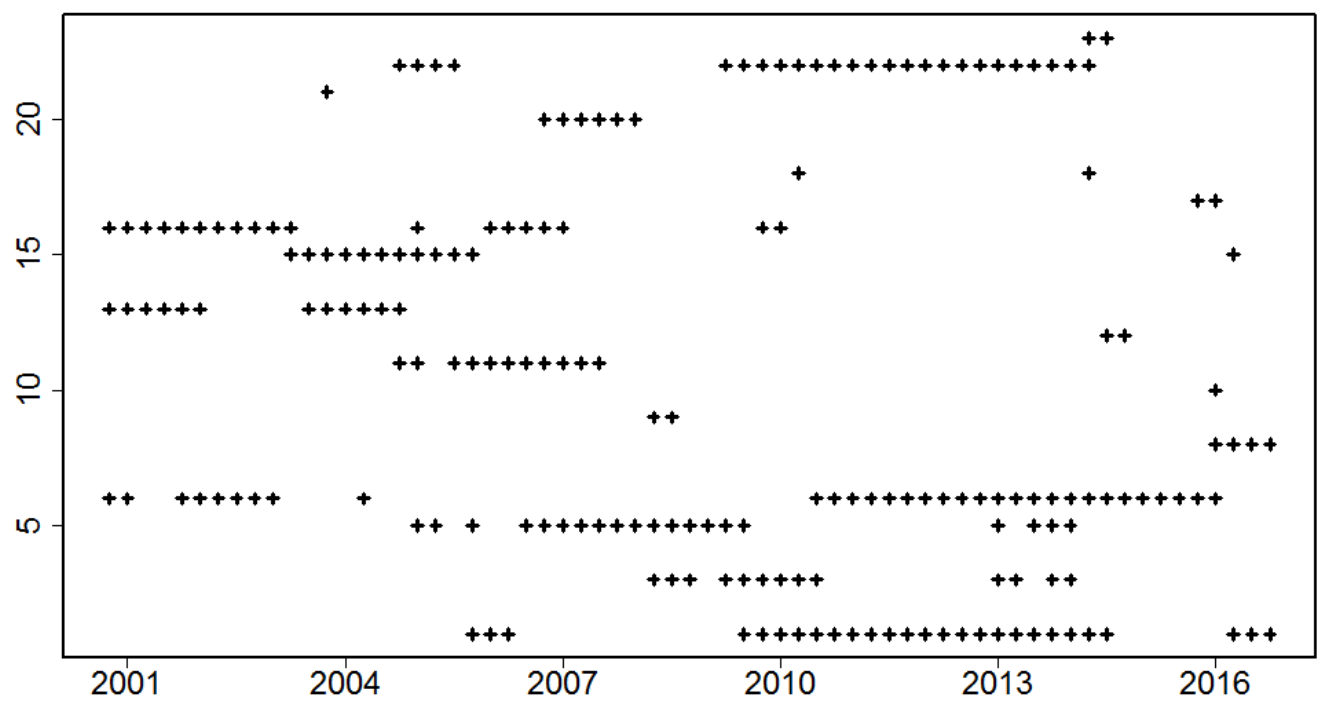

Notes: The $\mathrm{x}$-axis denotes time, and the $\mathrm{y}$-axis denotes forecaster ranking, where a smaller y-axis location refers to a forecaster with smaller overall RMSE. A "+" symbol at location $(x, y)$ indicates that forecaster $y$ was selected at time $x$.

average performers, and some ex post poor performers. Related, the ex-post best forecaster (ID 1) is not always selected, and conversely, the ex-post worst forecasters (ID's 22 and 23) are sometimes selected, mostly toward the end of the sample following the Great Recession.

Second, the selected set is not dominated by any one forecaster, or a small set of forecasters. Different forecasters move in and out of the selected set as we roll through the sample. ${ }^{12}$ This may be due to different forecasters having different skills, which are relevant at different times. Some may be better in recessions and some in recoveries, some may have more insights into macro-finance interactions, etc. ${ }^{13}$

Finally, the selected set, although evolving, is not at all independent over time; that is, the forecasters are not exchangeable. If a forecaster is in the selected set at time $t$, it is highly likely that she will be in the selected set at time $t+1$. This is evident from the many "horizontal streaks" in Figure 2.

\footnotetext{
${ }^{12}$ The most frequently selected forecasters are ID 6 (32 of 65 quarters), ID 1 (27 of 65 quarters), and ID 22 (25 of 65 quarters). Five forecasters are never selected: ID's 2, 4, 7, 14, and 19.

${ }^{13}$ Note, for example, the long streaks of ID's 1, 6, and 22 immediately following the Great Recession.
} 


\section{Sophisticated Averaging Inspired by the Ex Post Optimal peLASSO Tuning}

Here, motivated by the structure of the ex post (infeasible) peLASSO solution, we propose and explore procedures that implement that structure directly (discard most forecasts, and then average the survivors), while eliminating the need for penalty parameter selection. Our procedures implicitly perform sophisticated forward-looking cross validation tailored precisely to the forecasting problem at hand, but again, with no need for penalty parameter selection.

\section{1 "Average Best" Combination}

Directly motivated by the ex post peLASSO solution, we select a small number $N$ of "best" forecasts and average them. There are two ways to do the selection - from an individual perspective and a portfolio perspective. We consider them in turn.

\subsubsection{Individual-Based Average-Best Combination}

At each time, rolling forward, we determine the best $N$ individual forecasters over the past 20 quarters, and then we average their 1-year-ahead forecasts. We refer to this as "individualbased average-best $N$ " forecast combination.

Average-best combination requires choosing $N$, and the results of course depend on $N$. As shown in Table 2, and as expected, for individual-based average-best there is an internal optimum (minimum) RMSE for small $N$ (3 or 4). The optimized RMSE, moreover, is highly competitive, much better than the ex post worst forecaster, noticeably better than the simple average, and indeed about as good as the ex post best forecaster. The DM statistics, however, are at best only borderline significant, presumably due to the very small forecast evaluation sample size, as was also the case for infeasible peLASSO.

There is a slight ex post aspect of the good performance of average-best $N$ forecasts, because the optimal $N$ is not known in ex ante. Instead of fixing $N$ arbitrarily, we can proceed as follows: At each time examine the historical performance of average-best $N$ for $N=1, \ldots, N_{\max }$, pick the best, and use that $N$ and those forecasters to produce the forecast. We refer to this as "individual-based average-best $\leq N_{\max }$ " forecast combination, and it also appears in Table 2. The RMSE's tend to drop with $N$, quickly asymptoting around $N=3$. 
Table 2: Individual-Based Average-Best Forecast Combination

\begin{tabular}{lcccc}
\hline \hline Average-Best $N$ & RMSE & $\#$ & DM & p-val \\
\hline$N=1$ & 1.46 & 1 & 0.33 & 0.37 \\
$N=2$ & 1.42 & 2 & 0.77 & 0.22 \\
$N=3$ & 1.41 & 3 & 0.84 & 0.20 \\
$N=4$ & 1.41 & 4 & 0.95 & 0.17 \\
$N=5$ & 1.42 & 5 & 1.09 & 0.14 \\
$N=6$ & 1.43 & 6 & 1.11 & 0.14 \\
\hline \hline Average-Best $\leq N_{\max }$ & $\mathrm{RMSE}$ & $\#$ & $\mathrm{DM}$ & $\mathrm{p}$-val \\
\hline$N_{\max }=1$ & 1.46 & 1.00 & 0.33 & 0.37 \\
$N_{\max }=2$ & 1.44 & 1.45 & 0.55 & 0.29 \\
$N_{\max }=3$ & 1.44 & 1.63 & 0.54 & 0.30 \\
$N_{\max }=4$ & 1.44 & 1.74 & 0.57 & 0.29 \\
$N_{\max }=5$ & 1.44 & 1.83 & 0.57 & 0.29 \\
$N_{\max }=6$ & 1.44 & 1.86 & 0.57 & 0.29 \\
\hline \hline Comparisons & $\mathrm{RMSE}$ & $\#$ & $\mathrm{DM}$ & $\mathrm{p}$-val \\
\hline Best & 1.40 & 1 & 0.61 & 0.27 \\
$90 \%$ & 1.44 & 1 & 0.63 & 0.27 \\
Median & 1.53 & 1 & -0.57 & 0.72 \\
$10 \%$ & 1.68 & 1 & -1.61 & 0.94 \\
Worst & 1.74 & 1 & -1.55 & 0.94 \\
\hline Average & 1.50 & 23 & $\mathrm{~N} / \mathrm{A}$ & $\mathrm{N} / \mathrm{A}$ \\
\hline \hline
\end{tabular}

Notes: \# is the average number of forecasters selected, DM is the one-sided Diebold and Mariano (1995) statistic against a simple average, and p-val is the associated p-value. We compute DM as in Harvey et al. (1999). 


\subsubsection{Portfolio(LASSO)-Based Average-Best Combination}

We have already noted the "trim and average" form of the ex post optimal peLASSO solution. It is important to note, however, that its trimming is sophisticated, insofar as peLASSO does not trim the worst forecasters from an individual perspective. Rather, peLASSO trims the worst forcasters from a portfolio perspective, that is, those forecasters with the least to contribute to the combined forecast. The two concepts are very different, and so far we have considered only the individual perspective. The portfolio perspective suggests a related but different portfolio-based average-best $N$ strategy: At each time, rolling forward, use the LASSO to determine the best $N$ forecasters over the relevant window, and then average their forecasts. We refer to this as "LASSO-based average-best $N$ " forecast combination. Results appear in Table 3, which also includes results for LASSO-based average-best $\leq N_{\max }$ combinations. Surprisingly, the LASSO-based average-best forecasts perform no better - in fact they are slightly worse - than the individual-based average-best forecasts.

\section{2 "Best Average" Combination}

In the "average best" approach above, at each time, rolling forward, we select some best forecasters and average their forecasts. Here we move to a "best average" approach, instead selecting directly over averages. At each time, rolling forward, we simply find the historically best-performing average, and use it. Best-average is the more direct approach.

A first strategy is "best $N$-Average". At each time we use a 20 -quarter window and determine the best-performing $N$-forecast average and use it. A second strategy is "best $\leq N_{\text {max }^{-}}$ Average". At each time we use a 20-quarter window and determine the best-performing $\leq N_{\max }$-forecast average and use it.

Best-average combining can involve significant computation depending on $K$ and $N$ or $N_{\max }$. For example, with 23 forecasters finding the best 6-average requires computing ${ }_{23} C_{6}$ $(=100,947)$ simple averages and then sorting them to determine the minimum, each period as we roll through time. The per-period computational burden of $\leq N_{\max }$-forecast averaging is still larger, because we now consider all subsets. For example, finding the best $\leq 6$-average with 23 forecasters requires computing ${ }_{23} C_{6}+{ }_{23} C_{5}+\ldots+{ }_{23} C_{1}(=145,498)$ simple averages and then sorting them to determine the minimum. Fortunately, the relevant $K$ and $N_{\max }$ are quite small in typical economic forecast combinations. In our case, for example, $K=23$, and $N_{\max } \leq 6$ appears more than adequate.

In Table 4 we show results for both best $N$-average combinations $(N=1, \ldots, 6)$ and best 
Table 3: LASSO-Based Average-Best Forecast Combination

\begin{tabular}{lcccc}
\hline \hline Average-Best $N$ & RMSE & $\#$ & DM & p-val \\
\hline$N=1$ & 1.56 & 1 & -1.59 & 0.94 \\
$N=2$ & 1.53 & 2 & -0.55 & 0.71 \\
$N=3$ & 1.45 & 3 & 0.87 & 0.19 \\
$N=4$ & 1.45 & 4 & 0.92 & 0.18 \\
$N=5$ & 1.46 & 5 & 0.86 & 0.20 \\
$N=6$ & 1.47 & 6 & 0.89 & 0.19 \\
\hline \hline Average-Best $\leq N_{\max }$ & RMSE & $\#$ & DM & p-val \\
\hline$N_{\max }=1$ & 1.56 & 1 & -1.59 & 0.94 \\
$N_{\max }=2$ & 1.50 & 1.82 & 0.14 & 0.45 \\
$N_{\max }=3$ & 1.47 & 2.35 & 0.55 & 0.29 \\
$N_{\max }=4$ & 1.47 & 2.51 & 0.54 & 0.29 \\
$N_{\max }=5$ & 1.47 & 2.57 & 0.57 & 0.29 \\
$N_{\max }=6$ & 1.47 & 2.57 & 0.57 & 0.29 \\
\hline \hline Comparisons & $\mathrm{RMSE}$ & $\#$ & $\mathrm{DM}$ & $\mathrm{p}-\mathrm{val}$ \\
\hline Best & 1.40 & 1 & 0.61 & 0.27 \\
$90 \%$ & 1.44 & 1 & 0.63 & 0.27 \\
Median & 1.53 & 1 & -0.57 & 0.72 \\
$10 \%$ & 1.68 & 1 & -1.61 & 0.94 \\
Worst & 1.74 & 1 & -1.55 & 0.94 \\
\hline Average & 1.50 & 23 & $\mathrm{~N} / \mathrm{A}$ & $\mathrm{N} / \mathrm{A}$ \\
\hline \hline
\end{tabular}

Notes: \# is the average number of forecasters selected, DM is the one-sided Diebold and Mariano (1995) statistic against a simple average, and p-val is the associated p-value. We compute DM as in Harvey et al. (1999). 
Table 4: Best-Average Forecast Combination

\begin{tabular}{lcccc}
\hline \hline Best $N$-Average & RMSE & $\#$ & DM & p-val \\
\hline$N=1$ & 1.46 & 1 & 0.33 & 0.37 \\
$N=2$ & 1.41 & 2 & 0.80 & 0.21 \\
$N=3$ & 1.42 & 3 & 0.78 & 0.22 \\
$N=4$ & 1.41 & 4 & 0.92 & 0.18 \\
$N=5$ & 1.42 & 5 & 1.11 & 0.13 \\
$N=6$ & 1.42 & 6 & 1.28 & 0.10 \\
\hline \hline Best $\leq N_{\text {max }}$-Average & RMSE & $\#$ & DM & p-val \\
\hline$N_{\max }=1$ & 1.46 & 1 & 0.33 & 0.37 \\
$N_{\max }=2$ & 1.44 & 1.52 & 0.61 & 0.27 \\
$N_{\max }=3$ & 1.44 & 1.72 & 0.60 & 0.27 \\
$N_{\max }=4$ & 1.44 & 1.80 & 0.60 & 0.28 \\
$N_{\max }=5$ & 1.44 & 1.83 & 0.61 & 0.27 \\
$N_{\max }=6$ & 1.44 & 1.83 & 0.61 & 0.27 \\
\hline \hline Comparisons & $\mathrm{RMSE}$ & $\#$ & $\mathrm{DM}$ & $\mathrm{p}$-val \\
\hline Best & 1.40 & 1 & 0.61 & 0.27 \\
$90 \%$ & 1.44 & 1 & 0.63 & 0.27 \\
Median & 1.53 & 1 & -0.57 & 0.72 \\
$10 \%$ & 1.68 & 1 & -1.61 & 0.94 \\
Worst & 1.74 & 1 & -1.55 & 0.94 \\
\hline Average & 1.50 & 23 & $\mathrm{~N} / \mathrm{A}$ & $\mathrm{N} / \mathrm{A}$ \\
\hline \hline
\end{tabular}

Notes: \# is the average number of forecasters selected, DM is the one-sided Diebold and Mariano (1995) statistic against a simple average, and p-val is the associated p-value. We compute DM as in Harvey et al. (1999). 
$\leq N_{\max }$-average combinations $\left(N_{\max }=1, \ldots, 6\right)$. For both variations, the optima are achieved for small $N$ or $N_{\max }$. One might expect best-average methods to outperform average-best, because best-average directly targets the object of interest. Although best-average does not outperform, it also does not underperform: It is at least as good as anything else. Best average $\leq 6$ RMSE is almost as good as that of the best individual, much better than that of the median individual, and importantly, better than that of the simple average.

\subsection{On Estimation Window Width}

The essence of the rolling best-average approach is simply to use the particular average that has performed best in the "recent" past. But there is of course no reason why the appropriate notion of "recent" (that is, the appropriate choice $W$ of the most-recent $W$ quarters for evaluation) should be $W=20$. Using a more complete notation, let us denote our earlier best $N$-average as best $(N, 20)$-average, to indicate both an $N$-forecast average and a 20-period evaluation window. Generically, then, we can speak of best $(N, W)$-average or best $\left(\leq N_{\max }, W\right)$-average combinations.

In the first panel of Table 5 we show results for best $\left(\leq N_{\max }, W\right)$-average combinations with $N_{\max }=6$ and $W$ ranging from 1 through 40 . The RMSE performance of the best $(\leq 6, W)$-average approach is relatively insensitive to $W$, but it is clearly optimized for very small $W$, around 2 or 3 . Interestingly, the average number of forecasters selected around the optimal $W$ is also very small $(N \approx 2)$. So the optimal procedure (best $(\leq 6,2)$-average) is very "localized" - each period it basically averages the two forecasts of the two forecasters who have performed best during the past two quarters. It has $R M S E$ better than the ex post best forecaster, and much better than the average forecaster, with a DM p-value of 0.07.

We can also allow for time-varying window width $W$; that is, we can work with best $\left(\leq N_{\max }, \leq W_{\max }\right)$-averages, which are completely ex ante. They turn out to work very well: The best $(\leq 6, \leq 40$ )-average (in the one-line middle panel of Table 5 ) has $R M S E$ better than the best forecaster, and much better than the average forecaster - with a DM p-value of 0.11 . All told, allowing for time-varying window width appears highly valuable.

\section{$5 \quad$ Related Literature}

Now that we have introduced our approach, we can relate it to certain aspects of the broader literature. 
Table 5: Forecast Combination

\begin{tabular}{lccccc}
\hline \hline $\begin{array}{l}\text { Best }(\leq 6, W) \\
\text {-Average }\end{array}$ & RMSE & $\# N$ & $\# W$ & DM & p-val \\
\hline$W=1$ & 1.42 & 1.14 & 1 & 1.14 & 0.13 \\
$W=2$ & 1.36 & 1.54 & 2 & 1.50 & 0.07 \\
$W=3$ & 1.37 & 1.45 & 3 & 1.41 & 0.08 \\
$W=4$ & 1.40 & 1.29 & 4 & 1.10 & 0.14 \\
$W=5$ & 1.42 & 1.41 & 5 & 0.93 & 0.18 \\
$W=6$ & 1.42 & 1.43 & 6 & 0.81 & 0.21 \\
$W=7$ & 1.44 & 1.43 & 7 & 0.65 & 0.26 \\
$W=8$ & 1.46 & 1.54 & 8 & 0.41 & 0.34 \\
$W=9$ & 1.47 & 1.70 & 9 & 0.37 & 0.36 \\
$W=10$ & 1.46 & 1.70 & 10 & 0.43 & 0.33 \\
$W=15$ & 1.44 & 1.77 & 15 & 0.66 & 0.26 \\
$W=20$ & 1.44 & 1.78 & 20 & 0.61 & 0.27 \\
$W=25$ & 1.46 & 1.57 & 25 & 0.40 & 0.34 \\
$W=30$ & 1.48 & 1.62 & 30 & 0.19 & 0.42 \\
$W=35$ & 1.48 & 1.67 & 35 & 0.29 & 0.39 \\
$W=40$ & 1.48 & 1.74 & 40 & 0.22 & 0.41 \\
\hline Best $(\leq 6, \leq 40)$ & & & & & \\
-Average & 1.38 & 1.38 & 2.02 & 1.24 & 0.11 \\
\hline \hline Comparisons & RMSE & $\# N$ & $\# W$ & DM & p-val \\
\hline Best & 1.40 & 1 & N/A & 0.61 & 0.27 \\
$90 \%$ & 1.44 & 1 & N/A & 0.63 & 0.27 \\
Median & 1.53 & 1 & N/A & -0.57 & 0.72 \\
$10 \%$ & 1.68 & 1 & N/A & -1.61 & 0.94 \\
Worst & 1.74 & 1 & N/A & -1.55 & 0.94 \\
\hline Average & 1.50 & 23 & N/A & N/A & N/A \\
\hline \hline
\end{tabular}

Notes: $\# N$ is the average number of forecasters selected, $\# W$ is the average window width selected, DM is the one-sided Diebold and Mariano (1995) statistic against a simple average, and p-val is the associated p-value. We compute DM as in Harvey et al. (1999). 


\subsection{On Selection}

The structure of the peLASSO solution, which motivates our direct average-best and bestaverage procedures, clearly involves harsh "trimming" - resulting in the elimination of most forecasters. Trimming has been used in forecast combination by many authors, such as Stock and Watson (1999), Aiolfi and Favero (2005), Aiolfi and Timmermann (2006), Bjørnland et al. (2012), and Genre et al. (2013). But as noted by Granger and Jeon (2004), the attractiveness of trimming may be "more of a pragmatic folk-view than anything based on a clear theory".

One can view our results as showing the clear emergence of the "folk view" in a framework rigorously based on a "clear theory". In particular, although the peLASSO solution in principle need not involve trimming (i.e., it is possible for the peLASSO solution to feature shrinkage but not selection), we have shown that in practice it does, and indeed that it involves heavy trimming. Interestingly, Samuels and Sekkel (2017) obtain the same result using a very different approach based on the "model confidence sets" of Hansen et al. (2011). Note, moreover, that both our trimming procedure (in peLASSO, LASSO-based average-best, and best-average) and that of Samuels and Sekkel (2017) are generally quite sophisticated insofar as they trim from a portfolio perspective rather than a stand-alone perspective. Most impressively, Conflitti et al. (2015) impose sum-to-one and non-negativity constraints, which lead to a sparse solution (that is, some of combination weights are exactly zero) with combining weights shrunken - indeed forced - to be in $[0,1]$, all of which is in close touch with the concerns of forecast combination. ${ }^{14}$

\subsection{On Shrinkage}

Several authors have considered Bayesian shrinkage of combining weights. As is well known, under standard conditions the Bayes rule under quadratic loss is

$$
\beta_{1}=\beta_{0}+\delta\left(\hat{\beta}_{O L S}-\beta_{0}\right)
$$

where $\beta_{1}$ is the posterior mean combining weight vector, $\beta_{0}$ is the prior mean vector, and $\delta \in[0,1]$ is inversely related to prior precision. Other things equal, a small value of $\delta$ implies high prior precision and hence substantial shrinkage toward $\beta_{0}$. The larger is $\delta$, the less shrinkage occurs. Different authors invoke different shrinkage directions (prior means) and different ways of choosing $\delta$. Relevant literature includes Diebold and Pauly (1990), Chan

\footnotetext{
${ }^{14}$ Their estimator can be shown to be a special case of LASSO.
} 
et al. (1999), Stock and Watson (2004), Aiolfi and Timmermann (2006), and Genre et al. (2013).

In an interesting development, Capistrán and Timmermann (2009) take a reverse approach. Whereas Bayesian shrinkage adjusts least-squares combining weights toward a simple average, Capistrán and Timmermann (2009) start with a simple average and adjust away from it via a Mincer-Zarnowitz regression, $y_{t} \rightarrow c, \bar{f}_{t}$.

\subsection{Relatives of peLASSO}

The reverse approach of Capistrán and Timmermann (2009) has an interesting connection to the so-called "OSCAR LASSO" proposed by Bondell and Reich (2008), which is also closely related to our methods.

First let us introduce OSCAR. It is defined by the penalized regression:

$$
\begin{gathered}
\hat{\beta}_{O S C A R}=\arg \min _{\beta} \sum_{t=1}^{T}\left(y_{t}-\sum_{i=1}^{K} \beta_{i} x_{i t}\right)^{2} \\
\text { s.t. }(1-\gamma) \sum_{i=1}^{K}\left|\beta_{i}\right|+\gamma \sum_{j<k} \max \left\{\left|\beta_{j}\right|,\left|\beta_{k}\right|\right\} \leq c .
\end{gathered}
$$

The first part of the constraint involves the $L_{1}$ norm; it is just the standard LASSO constraint, producing selection and shrinkage toward zero. The second part of the constraint involves the pairwise $L_{\infty}$ norm, which selects and shrinks toward equal coefficients. Overall, then, OSCAR regression encourages parsimony not only in standard LASSO fashion, but also by encouraging a small number of unique nonzero coefficients on surviving covariates. ${ }^{15}$

Now let us link to Capistrán and Timmermann (2009). Suppose that the OSCAR solution is "all coefficients are the same". This can occur because of the second part of the OSCAR constraint. Then the combined forecast is

$$
\begin{aligned}
\hat{C}_{t} & =\hat{\beta} \sum_{i}^{K} f_{i, t} \\
& =\hat{\alpha}\left(\frac{1}{K} \sum_{i=1}^{K} f_{i, t}\right),
\end{aligned}
$$

\footnotetext{
${ }^{15}$ Note, however, that although OSCAR shrinks toward "equal weights", the equal weights need not correspond to simple averages (e.g., each of three selected forecasters might get weight $1 / 2$ ). This is potentially very important in our context.
} 
which is the forecast we get by projecting the realized outcome on equal-weight forecasts, as in Capistrán and Timmermann (2009). The OSCAR solution may also have more than one unique coefficient. In particular, it may have multiple groups, as for example with

$$
\begin{aligned}
\hat{C}_{t} & =\hat{\beta}_{1} \sum_{i \in G_{1}} f_{i, t}+\hat{\beta}_{2} \sum_{i \in G_{2}} f_{i, t} \\
& =\hat{\alpha}_{1}\left(\frac{1}{N_{1}} \sum_{i \in G_{1}} f_{i, t}\right)+\hat{\alpha}_{2}\left(\frac{1}{N_{2}} \sum_{i \in G_{2}} f_{i, t}\right),
\end{aligned}
$$

where $G_{k}=\left\{i: \hat{\beta}_{i}=\hat{\beta}_{k}\right\}$ and $N_{k}$ is the size of group $G_{k}$. The approaches of Aiolfi and Timmermann (2006) and Genre et al. (2013), which allow for grouping, are in the same spirit, as are the "homogeneity pursuit" procedure of Ke et al. (2015) and the "HORSES" procedure of Jang et al. (2015).

\subsection{Relatives of Average-Best and Best-Average}

Burgi and Sinclair (2017) is related to our average-best approach, essentially amounting to a refinement of our "individual" average-best. They proceed as follows: (1) For each forecaster, calculate a variable that takes a value of 1 in a given period if that forecaster has a lower squared error in that period than the simple average and 0 otherwise ${ }^{16}$; (2) If a forecaster beats the simple average more often than a percentage threshold $p$, include that forecaster in the selected subset for the next forecasting period; (3) Average over the selected forecasters.

The work most closely related to ours, however, is the seminal and to our knowledge relatively-unknown work of Elliott (2011), who examines gains from optimal combination relative to simple averaging, provides conditions under which the two are equivalent, and explores aspects of what he calls "best subset averaging". Effectively we provide a foundation for Elliott's subset-averaging procedures, which initially appears ad hoc in theory, even if highly effective in practice. That is, we show that Elliott's procedures are not ad hoc in theory.

\footnotetext{
${ }^{16}$ The time-average of this variable is the historical percentage share of times that the forecaster has beaten the average.
} 


\section{Concluding Remarks}

Against a background of frequently-found superiority of simple-average forecast combinations, we have proposed "partially egalitarian LASSO" (peLASSO) procedures that discard some forecasts and then select and shrink - without forcing - those remaining toward equal weights. We found that the peLASSO solution involves discarding most forecasters and simply averaging the survivors. We therefore proposed alternative direct combination procedures, most notably "best average" combinations, and we showed that they appear highly competitive for out-of-sample forecasting. In particular, they often dominate simple averages in forecasting Eurozone GDP growth.

A key insight is that the structure and success of our averaging procedures are entirely motivated by and consistent with the lessons learned from peLASSO. Among other things, we learn from peLASSO that (1) The selection penalty should be quite harsh - only a few forecasts need be combined, (2) The forecasts selected for combination should be regularized via shrinkage, (3) The shrinkage direction should be toward a simple average, not toward zero, or anything else, and (4) The shrinkage should be extreme, so that the selected forecasts should simply be averaged. All of this is embedded in our best $\leq\left(N_{\max }, W_{\max }\right)$-average procedure, for small $N_{\max }$ and $W_{\max }$.

\section{Appendices}

\section{A Egalitarian LASSO and Egalitarian Ridge Imple- mentation}

Egalitarian LASSO can be implemented via a straightforward adaptation of standard LASSO software, such as the R package glmnet, written by J. Friedman, T. Hastie, N. Simon, and R. Tibshirani, at https://cran.r-project.org/web/packages/glmnet/index.html. Simply note that

$$
\sum_{t=1}^{T}\left(y_{t}-\sum_{i=1}^{K} \beta_{i} f_{i t}\right)^{2}+\lambda \sum_{i=1}^{K}\left|\beta_{i}-\frac{1}{K}\right|=\sum_{t=1}^{T}\left(y_{t}-\bar{f}_{t}+\bar{f}_{t}-\sum_{i=1}^{K} \beta_{i} f_{i t}\right)^{2}+\lambda \sum_{i=1}^{K}\left|\beta_{i}-\frac{1}{K}\right|
$$




$$
\begin{gathered}
=\sum_{t=1}^{T}\left(\left(y_{t}-\bar{f}_{t}\right)+\sum_{i=1}^{K}\left(\frac{1}{K}-\beta_{i}\right) f_{i t}\right)^{2}+\lambda \sum_{i=1}^{K}\left|\beta_{i}-\frac{1}{K}\right| \\
=\sum_{t=1}^{T}\left(\left(y_{t}-\bar{f}_{t}\right)-\sum_{i=1}^{K} \delta_{i} f_{i t}\right)^{2}+\lambda \sum_{i=1}^{K}\left|\delta_{i}\right|,
\end{gathered}
$$

where

$$
\delta_{i}=\beta_{i}-\frac{1}{K} \quad \text { and } \quad \bar{f}_{t}=\frac{1}{K} \sum_{i=1}^{K} f_{i t} .
$$

Hence we obtain the egalitarian LASSO regression,

$$
y_{t} \rightarrow_{\text {EgalLASSO }} f_{1 t}, \ldots, f_{K t},
$$

by simply running the standard LASSO regression,

$$
\left(y_{t}-\bar{f}_{t}\right) \rightarrow_{L A S S O} f_{1 t}, \ldots, f_{K t} .
$$

Similarly, egalitarian ridge can be trivially implemented by $\left(y_{t}-\bar{f}_{t}\right) \rightarrow_{\text {Ridge }} f_{1 t}, \ldots, f_{k t}$, in precise parallel with egalitarian LASSO implementation. 


\section{B Equal-Length, 20-Quarter, Evaluation Windows}

Here we start from $t=21$ rather than $t=6$ when evaluating forecasts; hence the estimation sample sizes are always of length 20. The results, reported in Table 6, are qualitatively identical to those reported in the main text, for which the estimation sample sizes grow until $t=21$, after which they are always of length 20 .

Table 6: Forecast RMSE's Based on Ex-Post Optimal $\lambda$ 's, Evaluation Starts at $t=21$

\begin{tabular}{lccccc}
\hline \hline Regularization Group & RMSE & $\lambda^{*}$ & $\#$ & DM & p-val \\
\hline Ridge & 1.60 & 2.29 & 23.00 & 0.65 & 0.26 \\
LASSO & 1.61 & 0.38 & 2.78 & 0.22 & 0.42 \\
\hline eRidge & 1.58 & 1.97 & 23.00 & 0.96 & 0.17 \\
eLASSO & 1.60 & 0.51 & 23.00 & 0.82 & 0.21 \\
\hline peLASSO (LASSO, Average) & 1.51 & 0.21 & 3.12 & 1.14 & 0.13 \\
peLASSO (LASSO, eRidge) & 1.50 & $(0.21,3.10)$ & 3.12 & 1.06 & 0.15 \\
peLASSO (LASSO, eLASSO) & 1.50 & $(0.21,0.51)$ & 3.12 & 1.03 & 0.16 \\
\hline \hline Comparisons & RMSE & $\lambda^{*}$ & $\#$ & DM & p-val \\
\hline Best & 1.49 & N/A & 1 & 0.76 & 0.23 \\
90\% & 1.54 & N/A & 1 & 0.93 & 0.18 \\
Median & 1.65 & N/A & 1 & -0.38 & 0.65 \\
$10 \%$ & 1.82 & N/A & 1 & -1.37 & 0.91 \\
Worst & 1.90 & N/A & 1 & -1.46 & 0.92 \\
\hline Average & 1.64 & N/A & 23 & N/A & N/A \\
\hline \hline
\end{tabular}

Notes: $\lambda^{*}$ is the ex-post optimal penalty parameter(s), \# is the average number of forecasters selected, and DM is the one-sided Diebold and Mariano (1995) statistic against a simple average, with p-value denoted p-val. We compute DM as in Harvey et al. (1999). 


\section{Adaptive Partially-Egalitarian LASSO}

We change the LASSO penalty from $\lambda \sum_{k=1}^{K}\left|\beta_{k}\right|$ to $\lambda \sum_{k=1}^{K} \frac{1}{|\hat{\beta}|^{1 / 3}}\left|\beta_{k}\right|$, where $\hat{\beta}$ is a preliminary consistent estimator, which we set to the Ridge regression estimate. Use of aLASSO improves the ex post performance of 2-step LASSO procedures, as can be seen in Table 7.

Table 7: RMSE's Based on Ex-Post Optimal $\lambda$ 's, Using Adaptive Lasso

\begin{tabular}{lccccc}
\hline \hline Regularization Group & RMSE & $\lambda^{*}$ & $\#$ & DM & p-val \\
\hline Ridge & 1.51 & 2.66 & 23.00 & -0.02 & 0.51 \\
LASSO & 1.46 & 0.80 & 2.09 & 0.22 & 0.41 \\
\hline eRidge & 1.49 & 1.97 & 23.00 & 0.15 & 0.44 \\
eLASSO & 1.50 & $\max$ & 23.00 & 0.55 & 0.29 \\
\hline peLASSO(aLASSO, Average) & 1.33 & 1.08 & 1.69 & 0.95 & 0.17 \\
peLASSO (aLASSO, eRidge) & 1.33 & $(1.08, \max )$ & 1.69 & 0.95 & 0.17 \\
peLASSO (aLASSO, eLASSO) & 1.33 & $(1.08, \max )$ & 1.69 & 0.95 & 0.17 \\
\hline \hline Comparisons & $\mathrm{RMSE}$ & $\lambda^{*}$ & $\#$ & $\mathrm{DM}$ & $\mathrm{p}$-val \\
\hline Best & 1.40 & $\mathrm{~N} / \mathrm{A}$ & 1 & 0.61 & 0.27 \\
$90 \%$ & 1.44 & $\mathrm{~N} / \mathrm{A}$ & 1 & 0.63 & 0.27 \\
Median & 1.53 & $\mathrm{~N} / \mathrm{A}$ & 1 & -0.57 & 0.72 \\
$10 \%$ & 1.68 & $\mathrm{~N} / \mathrm{A}$ & 1 & -1.61 & 0.94 \\
Worst & 1.74 & $\mathrm{~N} / \mathrm{A}$ & 1 & -1.55 & 0.94 \\
\hline Average & 1.50 & $\mathrm{~N} / \mathrm{A}$ & 23 & $\mathrm{~N} / \mathrm{A}$ & $\mathrm{N} / \mathrm{A}$ \\
\hline \hline
\end{tabular}

Notes: $\lambda^{*}$ is the ex-post optimal penalty parameter(s), \# is the average number of forecasters selected, and DM is the one-sided Diebold and Mariano (1995) statistic against a simple average, with p-value denoted p-val. We compute DM as in Harvey et al. (1999). 


\section{References}

Aiolfi, M. and C.A. Favero (2005), "Model Uncertainty, Thick Modelling and the Predictability of Stock Returns," Journal of Forecasting, 24, 233-254.

Aiolfi, M. and A. Timmermann (2006), "Persistence in Forecasting Performance and Conditional Combination Strategies," Journal of Econometrics, 135, 31-53.

Arora, S., E. Hazon, and S. Kale (2012), "The Multiplicative Weights Update Method: A Meta-Algorithm and Applications," Theory of Computing, 8, 121-164.

Aruoba, S.B., F.X. Diebold, J. Nalewaik, F. Schorfheide, and D. Song (2012), "Improving GDP Measurement: A Forecast Combination Perspective," In X. Chen and N. Swanson (eds.), Recent Advances and Future Directions in Causality, Prediction, and Specification Analysis: Essays in Honour of Halbert L. White Jr., Springer, 1-26.

Bates, J.M. and C.W.J Granger (1969), "The Combination of Forecasts," Operations Research Quarterly, 20, 451-468.

Bjørnland, H., K. Gerdrup, A. Jore, C. Smith, and L. Thorsrud (2012), "Does Forecast Combination Improve Norges Bank Inflation Forecasts?" Oxford Bulletin of Economics and Statistics, 74, 163-179.

Bondell, H.D. and B.J. Reich (2008), "Simultaneous Regression Shrinkage, Variable Selection, and Supervised Clustering of Predictors with OSCAR," Biometrics, 64, 115-123.

Burgi, C. and T. Sinclair (2017), "A Nonparametric Approach to Identifying a Subset of Forecasters that Outperforms the Simple Average," Empirical Economics, 53, 101-115.

Capistrán, C. and A. Timmermann (2009), "Forecast Combination with Entry and Exit of Experts," Journal of Business and Economic Statistics, 27, 428-440.

Chan, Y.L., J.H. Stock, and M.W. Watson (1999), "A Dynamic Factor Model Framework for Forecast Combination," Spanish Economic Review, 1, 91-121.

Clemen, R.T. (1989), "Combining Forecasts: A Review and Annotated Bibliography (With Discussion)," International Journal of Forecasting, 5, 559-583.

Conflitti, C., C. De Mol, and D. Giannone (2015), "Optimal Combination of Survey Forecasts," International Journal of Forecasting, 31, 1096-1103. 
Diebold, F.X. (1989), "Forecast Combination and Encompassing: Reconciling Two Divergent Literatures," International Journal of Forecasting, 5, 589-592.

Diebold, F.X. and J.A. Lopez (1996), "Forecast Evaluation and Combination," In G.S. Maddala and C.R. Rao (eds.), Handbook of Statistics, North-Holland, 241-268.

Diebold, F.X. and R. Mariano (1995), "Comparing Predictive Accuracy," Journal of Business and Economic Statistics, 13, 253-365.

Diebold, F.X. and P. Pauly (1990), "The Use of Prior Information in Forecast Combination," International Journal of Forecasting, 6, 503-508.

Elliott, G. (2011), "Averaging and the Optimal Combination of Forecasts," Manuscript, Department of Economics, UCSD.

Elliott, G. and A. Timmermann (2016), Economic Forecasting, Princeton University Press.

Genre, V., G. Kenny, A. Meyler, and A. Timmermann (2013), "Combining Expert Forecasts: Can Anything Beat the Simple Average?" International Journal of Forecasting, 29, 108121.

Granger, C.W.J. and Y. Jeon (2004), "Thick Modeling," Empirical Economics, 21, 323-343.

Granger, C.W.J. and R. Ramanathan (1984), "Improved Methods of Combining Forecasts," Journal of Forecasting, 3, 197-204.

Hansen, P.R., A. Lunde, and J.M. Nason (2011), "The Model Confidence Set," Econometrica, 79, 453-497.

Harvey, D., S. Leybourne, and P. Newbold (1999), "Testing the Equality of Prediction Mean Squared Errors," International Journal of Forecasting, 13, 281-291.

Hastie, T., R. Tibshirani, and J. Friedman (2009), The Elements of Statistical Learning, Springer.

Jang, W., J. Lim, N.A. Lazar, J.M. Loh, and D. Yu (2015), "Some Properties of Generalized Fused Lasso and Its Applications to High Dimensional Data," Journal of the Korean Statistical Society, 44, 352-365.

Ke, Z.T., J. Fan, and Y. Wu (2015), "Homogeneity Pursuit," Journal of the American Statistical Association, 110, 175-194. 
Samuels, J.D. and R.M. Sekkel (2017), "Model Confidence Sets and Forecast Combination," International Journal of Forecasting, 33, 48-60.

Smith, J. and K.F. Wallis (2009), "A Simple Explanation of the Forecast Combination Puzzle," Oxford Bulletin of Economics and Statistics, 71, 331-355.

Stock, J.H. and M.W. Watson (1999), "A Comparison of Linear and Nonlinear Univariate Models for Forecasting Macroeconomic Time Series," Chapter 1 of R.F. Engle and H. White (eds.), Cointegration, Causality, and Forecasting, Oxford University Press.

Stock, J.H. and M.W. Watson (2004), "Combination Forecasts of Output Growth in a SevenCountry Data Set," Journal of Forecasting, 23, 405-430.

Tibshirani, R. (1996), "Regression Shrinkage and Selection via the LASSO," Journal of the Royal Statistical Society, Series B, 267-288.

Timmermann, A. (2006), "Forecast Combinations," Handbook of Economic Forecasting, 135196.

Zellner, A. (1986), "On Assessing Prior Distributions and Bayesian Regression Analysis with g-Prior Distributions," in P.K. Goel and A. Zellner (eds.), Bayesian Inference and Decision Techniques: Essays in Honor of Bruno De Finetti, 233-243.

Zou, H. (2006), "The Adaptive LASSO and Its Oracle Properties," Journal of the American Statistical Association, 101, 1418-1429.

Zou, H. and T. Hastie (2005), "Regularization and Variable Selection via the Elastic Net," Journal of the Royal Statistical Society, Series B (Statistical Methodology), 67, 302-320. 